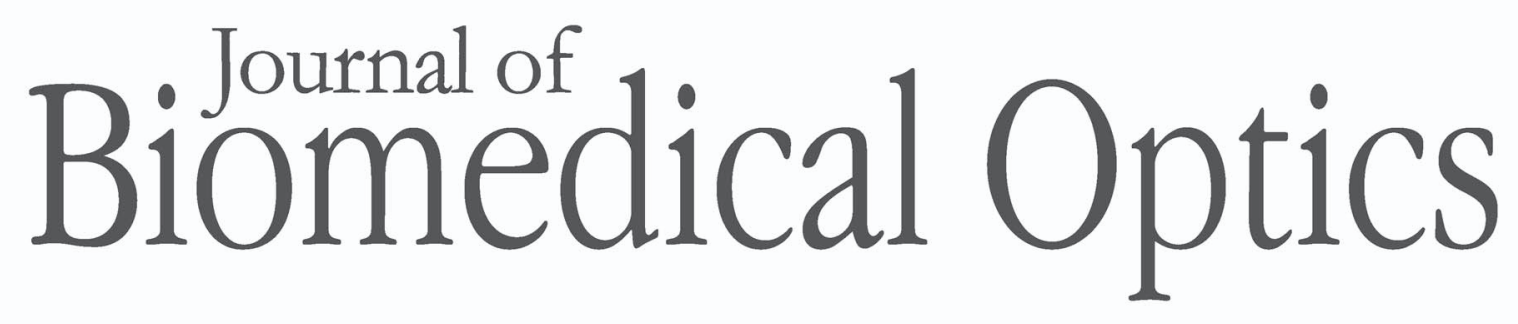

\title{
Optical coherence tomography to investigate optical properties of blood during coagulation
}

Xiangqun $\mathrm{Xu}$

Jia Lin

Feifei Fu 


\title{
Optical coherence tomography to investigate optical properties of blood during coagulation
}

\author{
Xiangqun Xu, Jia Lin, and Feifei Fu \\ Zhejiang Sci-Tech University, School of Science, Hangzhou 310018, China
}

\begin{abstract}
This study investigates the optical properties of human blood during the coagulation process under statics using optical coherence tomography (OCT). OCT signal slope (OCTSS) and 1/e light penetration depth $\left(d_{1 / \mathrm{e}}\right)$ were obtained from the profiles of reflectance versus depth. Results showed that both OCTSS and $d_{1 / \mathrm{e}}$ were able to sensitively differentiate various stages of blood properties during coagulating. After $1 \mathrm{~h}$ clotting, OCTSS decreased by $47.0 \%, 15.0 \%, 13.7 \%$, and $8.5 \%$ and $d_{1 / e}$ increased by $34.7 \%, 29.4 \%, 24.3 \%$, and $22.9 \%$ for the blood samples at HCT of $25 \%, 35 \%, 45 \%$, and $55 \%$, respectively. The slope of $d_{1 / \text { e }}$ versus time $\left(S_{r}, \times 10^{-4}\right.$ $\mathrm{mm} / \mathrm{s}$ ), associated with clot formation rate decreased from $6.0 \pm 0.3,3.7 \pm 0.5$ to $2.3 \pm 0.4$ with the increasing of HCT from $35 \%, 45 \%$, to $55 \%$. The clotting time $\left(t_{\mathrm{c}}\right)$ from the $d_{1 / \mathrm{e}}$ evolution curves was estimated to be 1969 $\pm 92 \mathrm{~s}, 375 \pm 12 \mathrm{~s}, 455 \pm 11 \mathrm{~s}$, and $865 \pm 47 \mathrm{~s}$ for the blood of $25 \%, 35 \%, 45 \%$, and $55 \%$. This study demonstrates that the parameters $\left(t_{C}\right.$ and $\left.S_{r}\right)$ from the variations in $d_{1 / e}$ had better sensitivity and smaller standard deviation. Furthermore, blood hematocrit affecting backscattering properties of blood during coagulation was capable of being discerned by OCT parameters. It is concluded that OCT is a potential technique to quantify and follow the liquid-gel transition of blood during clotting. @ 2011 Society of Photo-Optical Instrumentation Engineers (SPIE). [DOI: 10.1117/1.3615667]
\end{abstract}

Keywords: optical coherence tomography; hematocrits; blood coagulation; optical coherence tomography signal slope; 1/e light penetration depth.

Paper 10435RRRR received Aug. 4, 2010; revised manuscript received Jun. 15, 2011; accepted for publication Jul. 6, 2011; published online Sep. 2, 2011.

\section{Introduction}

Blood coagulation is a natural process that can be both beneficial and life-threatening for the human body. It allows the maintenance of hemostasis after vascular injury, but it can also cause deep vein thrombosis and heart stroke. Inappropriate blood coagulation represents the leading cause of morbidity and mortality in the United States, accounting for roughly $40 \%$ of all deaths. ${ }^{1}$ Most existing clinical tests of hemostasis such as the prothrombin time, partial thromboplastin, and activated clotting time tests, are performed on plasma containing all the coagulation factors. They are assays designed to identify specific defects in clot formation, but cannot identify hypercoagulability. These assays are limited by the variability in reagents, methodologies, and artificial conditions of the assay that do not precisely reproduce the physiologic event. Furthermore, they do not provide any information about the quality of the clot or the dynamics of its formation. ${ }^{2,3}$ During the last decades, many disciplines of applied research have tried to understand the complex phenomenon of the blood clotting process, namely mechanical, optical, and ultrasound characterization methods for in vitro tests. The viscosity and elasticity detection methods produce variable results depending on the shape of the fibrin clumps, i.e., the quantity or viscosity of the fibrin and on the strength of the magnetic field of the element. ${ }^{4-6}$ Furthermore, it is impossible to detect coagulation unless the viscosity increases to above a specific level. The turbidity detection method involves mixing the plasma specimen and coagulation reagent, and then measuring changes in the transmitted light or the scattered light during the process of the

Address all correspondence to: Xiangqun Xu, Zhejiang Sci-Tech University, School of Science, No. 2 Street, Xiasha, Hangzhou, Zhejiang 310018 China. Tel: : 008613777833176; Fax: 008657187055836; E-mail: jadexu@163.com. plasma coagulating. ${ }^{7}$ Small changes in the transmitted or scattered light can be detected even with a low level of fibrin, ${ }^{8}$ and it is hence free from the shortcoming of the viscosity detection method. Nevertheless, few in vitro tests that are also used in clinical practice are performed on blood samples. Recent studies have revealed an important need to develop accurate and global standard in vitro coagulation tests using blood samples. ${ }^{9,10}$ These new tests should complement existing standard tests performed with plasma samples. Due to the nature of acoustic waves, ultrasound measurement techniques have the major advantages of allowing the use of blood samples. Measurements of ultrasound backscattering, sound velocity, and ultrasound attenuation have been used to analyze the process of blood coagulation and clot formation. ${ }^{11-14}$ In optics, blood coagulation was studied in a special beaker by analyzing the properties of the reflected waves. The blood samples were illuminated with a yttrium aluminum garnet laser and coagulation was characterized from the refractive index and absorption coefficient. ${ }^{15-17}$

For more than a decade, another growing field of research that allows to assess the microstructures of soft biologic tissues has been in development, namely optical coherence tomography (OCT), which relies on short temporal coherence interferometry and measures the optical path and intensity of back-reflected near-infrared light. ${ }^{18}$ Recently, the blood properties have been widely studied using OCT and Doppler OCT (ODT) due to their high resolution, real time, and noninvasive capabilities. ${ }^{19-22}$ OCT backscattering signal and ODT variance imaging has been extensively utilized in characterizing some properties of blood, such as hematocrit (HCT), red blood cell (RBC) aggregation,

1083-3668/2011/16(9)/096002/7/\$25.00 (C) 2011 SPIE 
blood optical clearing, and blood photocoagulation in ours and other groups. ${ }^{17,23-30}$

Because the OCT technique measures in-depth intensity distribution with high resolution, changes in the in-depth distribution of the blood-scattering coefficient or refractive index are reflected in changes in the OCT signal. ${ }^{24,27,28}$ Thus, because blood coagulation may introduce local changes in their optical properties (scattering coefficient and local and mean refractive indices), one can monitor and quantify the coagulation process by analyzing the OCT signal slope (OCTSS $)^{31}$ that reflects the least scattering of blood to some degree and $1 / e$ light penetration depth $\left(d_{1 / e}\right)^{32}$ that more accurately represents multiple scattering of blood. To better assess properties of blood during coagulation, the OCT signal slope and 1/e light penetration depth were attempted to be applied to follow the process of blood coagulation and clot formation from blood samples of various hematocrits ranging from $25 \%$ to $55 \%$. Measurements were performed using a $1310 \mathrm{~nm}$ OCT. The effect of hematocrit associated with blood properties during coagulating was investigated and discussed. The main purpose of the present study was to investigate the feasibility of OCT to provide a description of the coagulation process of human blood.

\section{Materials and Methods}

\subsection{Materials}

The fresh whole blood was taken from healthy volunteers aged from 22 to 27 yrs. The blood sample was stabilized by $3.2 \%$ sodium citrate solution with the volume ratio of 9:1 for anticoagulation purpose. The whole blood was centrifuged to separate the erythrocytes from the plasma. The concentrated erythrocytes were washed twice with a phosphate buffer $(\mathrm{pH}=7.4)$. The blood samples with various HCTs ranging from $25 \%$ to $55 \%$ were obtained by reconstituting the concentrated erythrocytes with the separated plasma. The coagulation process was initiated by adding calcium chloride $(0.25 \mathrm{M}$ with the volume of 1/9). All measurements were carried out within $12 \mathrm{~h}$ after the blood collection.

\subsection{OCT measurements}

A delay line OCT system with a $1310 \mathrm{~nm}$ central wavelength and $40 \mathrm{~nm}$ bandwidth light source $(18 \mathrm{~mW})$ was used. The light source yields an $18 \mu \mathrm{m}$ axial resolution in free space, or approximately $13 \mu \mathrm{m}$ within tissue if the mean refractive index of bulk tissue is assumed to be 1.38; this determines the axial imaging resolution of the system. The transverse resolution was measured at $15 \mu \mathrm{m}$. For the scanning speed of the reference arm at $100 \mathrm{~A}$-scans, an OCT image of $400 \times 100$ pixel size requires about 2 s. ${ }^{32,33}$

Experiment 1 was performed to measure the OCTSS and $d_{1 / e}$ of the reconstituting blood samples during coagulation. A rectangular glass cuvette of $\sim 5 \mathrm{~mm}$ height (thickness) and $10 \mathrm{~mm} \times 40 \mathrm{~mm}$ area was used as a blood sample holder. ${ }^{21}$ The sample holder was placed almost perpendicular to the probing beam, but tilted at a small angle of $\sim 5 \mathrm{deg}$, relative to the cell surface to ensure that only light scattered or reflected by blood constituents was detected and the specular reflection that occurs at the glass-fluid interface was eliminated from the detection

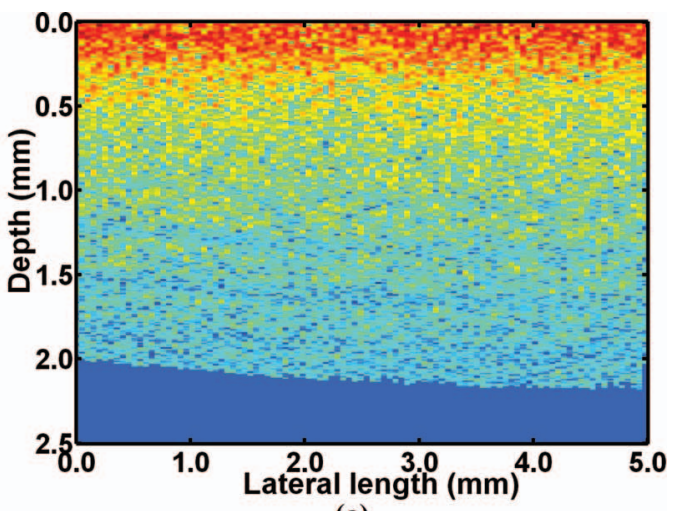

(a)

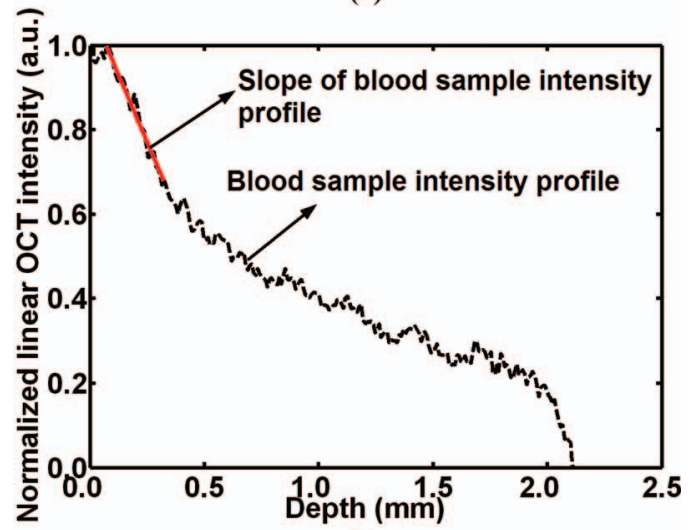

(b)

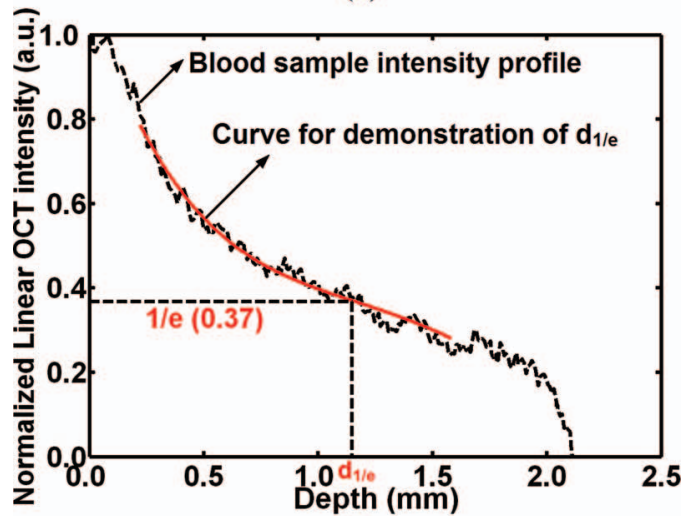

(c)

Fig. 1 An example of OCT data processing. (a) Digital registration of the tilt front surface of OCT scans by conducting an edge-detection algorithm to obtain a flat surface, (b) the linear fit for OCT signal slope extraction, and (c) the curve fit for 1/e light penetration depth extraction.

system. ${ }^{34}$ When the blood sample was added with $\mathrm{CaCl}_{2}$ solution as a coagulation agent, the OCT imaging of the blood sample was acquired immediately. The OCT images were taken every $\sim 12 \mathrm{~s}$, until $60 \mathrm{~min}$ after sampling. A cross-section imaging was obtained by taking $100 \mathrm{~A}$-scans over the lateral length of $5 \mathrm{~mm}$ and the axial depth of $2.5 \mathrm{~mm}$.

Experiment 2 was carried out to measure changes in refractive index of the plasma separated from the whole blood during coagulation. A glass tube with an inner diameter of $0.3 \mathrm{~mm}$ was used as a sample container. OCT images were taken every $5 \mathrm{~min}$ for a total of $30 \mathrm{~min}$ after sampling.

Data processing was illustrated in Fig. 1. Digital registration of the tilted blood surface because of the tilt cuvette in the OCT 

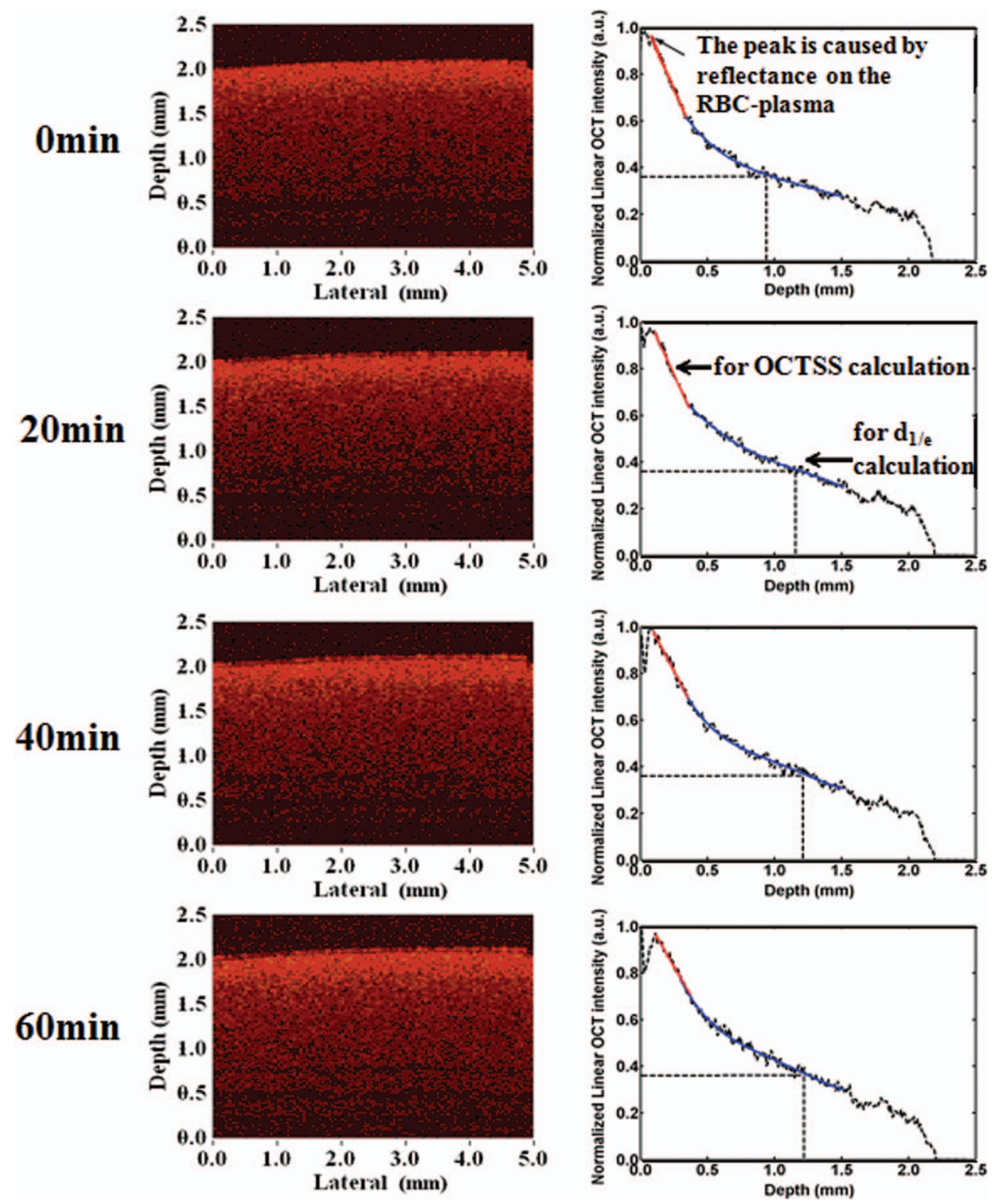

Fig. 2 OCT images (a) and corresponding OCT in-depth reflectance profiles (b) recorded from the blood sample at HCT $=45 \%$ at $0,20,40$, and 60 min during coagulation.

profiles based on an edge-detection algorithm was conducted to obtain a flat surface [Fig. 1(a)]. Quantitative data were obtained by averaging the signal intensity across the lateral imaging range as a function of depth. Every $100 \mathrm{~A}$ (depth) scans (covering the lateral length of $5 \mathrm{~mm}$ ) were averaged to one depth profile in the OCT imaging. The OCTSS was obtained by linear fitting of the region from the blood surface to the depth of $0.27 \mathrm{~mm}$ of the averaged depth profile. ${ }^{21,24,27,28}$ The region in the blood, where the signal was linear and underwent minimal alterations, was selected as shown in Fig. 1(b). A best fit exponential curve in depth was applied to the averaged and normalized signal from which the corresponding $1 / e$ light penetration depth was derived $^{32,33}$ [Fig. 1(c)]. The group refractive index of plasma was obtained from the equation $n_{g}=z_{\text {opt }} / z_{\text {phy }}$. In this study, the physical length $\left(z_{\text {phy }}\right)$ of all samples equals $\sim 0.3 \mathrm{~mm}$, i.e., the thickness of the sample container, which was determined by OCT. The optical path length $\left(z_{\mathrm{opt}}\right)$ of the plasma samples is measured by subtracting the axial position of the lower interface and that of the upper interface between the sample and the glass tube. $^{24}$

\section{Results and Discussion}

Figure 2 shows the representative OCT images [Fig. 2(a)] and the corresponding OCT in-depth reflectance profiles [Fig. 2(b)] recorded from the blood sample of a 26-year-old woman with HCT of $45 \%$ at $0,20,40$, and 60 min after coagulation. There was slight movement of the boundary between blood plasma and RBCs layers with time [Fig. 2(b)]. The sedimentation rate of $0.06 \pm 0.02 \mathrm{~mm} / \mathrm{h}$ for the clotting blood after $60 \mathrm{~min}$ coagulation was much smaller than that for the control $(2.16 \pm 0.11)$. The latter fits well the normal sedimentation rates for women ( 2 to $15 \mathrm{~mm} / \mathrm{h}$ ). The fibrin formed from fibrinogen in the blood plasma during blood coagulation may impede the RBC 


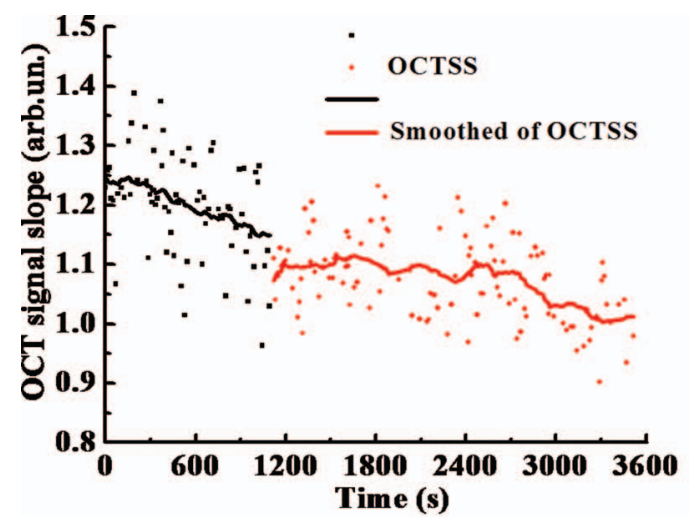

(a)

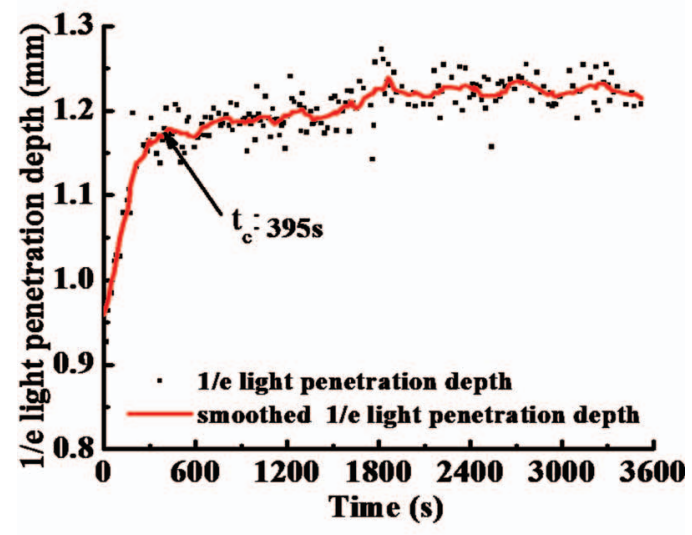

(b)

Fig. 3 Typical results for (a) OCT signal slope and (b) 1/e light penetration depth as a function of time for the blood sample at HCT $=35 \%$ during blood coagulation. The OCT signal slope decreased with time. The penetration depth increased accordingly and tended to a slow increase with a horizontal asymptote.

sedimentation. Normal blood sedimentation might cause a problem for accurate measurements of blood coagulation because sedimentation might change the optical properties as well, such as the increase of the 1/e light penetration depth.

Thanks to the slight sedimentation property of the blood during coagulation, we could accurately calculate the OCT signal slope and relative 1/e light penetration depth of the blood samples from the OCT in-depth reflectance profiles with the algorithm used in our previous works of Refs. 24, 27, 28, 31, 32, and 33. Figures 3(a) and 3(b), respectively, present the typical evolution of the OCTSS and $d_{1 / e}$ as a function of time acquired from the blood sample at HCT of $35 \%$ taking as an example. The mean OCTSS tended to decrease from $1.20 \pm 0.03$ to $1.04 \pm 0.06$ after $60 \mathrm{~min}$ coagulation. The entire process of blood coagulation relative to variations of the OCTSS, shown in Fig. 3(a) was divided into two stages. The results indicated that the OCTSS decreased faster during the first stage (0 to $1100 \mathrm{~s}$ ) than during the second stage (1100 to $3600 \mathrm{~s}$ ). We noted that the OCTSS measurement was not very accurate (large variation) by the time-domain OCT system. We expect better accuracy with a frequency domain OCT system that is able to reduce effectively the random noise. ${ }^{27,28}$ Nevertheless, the decreased OCTSS with blood coagulation was found.

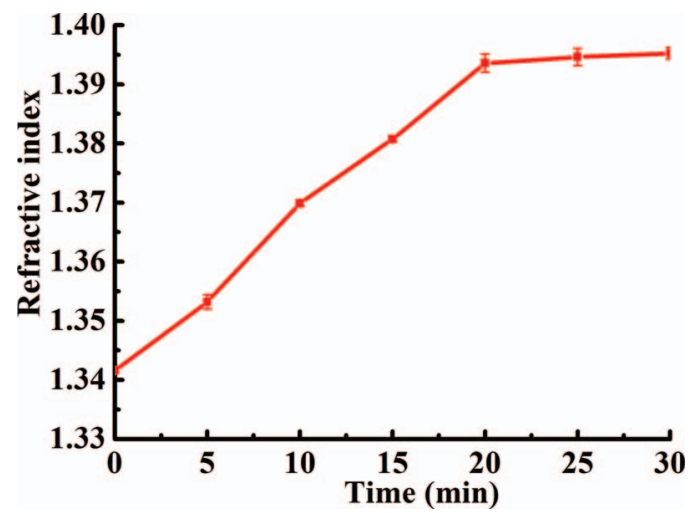

Fig. 4 Time-course changes in refractive index of plasma during coagulation. The refractive index increased accordingly and then changed a little after $20 \mathrm{~min}$.

The variations in $d_{1 / e}$ were represented by convex curve characterized by two stages [Fig. 3(b)]. The first stage corresponded to a rapid increase in penetration depth, and the second stage corresponded to a very slow increase with a horizontal asymptote. The curves of the variations in the penetration depth can be linked to changes in state of the medium and coincide with the two stages of a liquid to a solid/gel state. Before $\mathrm{CaCl}_{2}$ solution was added in the blood sample, the medium was in a liquid state. During stage 1 ( 0 to $395 \mathrm{~s}$ ), the medium is turning into a gel state as the transformation of fibrinogen into fibrin through a coagulation cascade reaction. In this stage, $d_{1 / e}$ increased rapidly. To better characterize variations of blood properties during coagulation and clot formation, the clotting time $\left(t_{c}\right)$ was defined as the period of time where 1/e light penetration depth curve started to be stabilized. For the blood of HCT of $35 \%$, the clotting time was estimated to be about $395 \mathrm{~s}$ [as indicated in an arrow in Fig. 3(b)]. In the following stage (396 to $3600 \mathrm{~s}$ ), variations in $d_{1 / e}$ were small.

Blood is a scattering system that consists of scattering particles, for example RBCs and the surrounding media, i.e., plasma. The refractive index mismatching between RBC cytoplasm and blood plasma is the major source of light scattering in blood. ${ }^{21}$ Following the addition of $\mathrm{CaCl}_{2}$ solution, a subsequent series of reactions including the conversion of plasma protein prothrombin into enzyme thrombin will be immediately initiated. Thrombin then catalyzes a reaction to allow several polypeptides to split from molecules of large rod-shaped plasma protein fibrinogen. The still-large fibrinogen remnants then bind to each other to form fibrin. During the blood coagulation process, the size, dimensions, and shape of the red blood cells almost do not change. The changes in the scattering resulted from the changes in its surrounding environment, i.e., plasma. During coagulation, the fibrinogen in the plasma transformed to coarse fibrin (the size from $1 \mathrm{~nm}$ to $1 \mu \mathrm{m}$ ). The refractive index of the plasma increased with the fibrin formation from 1.34 to 1.40 (Fig. 4). The refractive index of the red blood cells was measured to be 1.42. The reduced refractive index mismatching between the red blood cells and the plasma led to the decrease in the blood scattering, thus to the decrease of OCTSS and the enhancement of the light penetration depth. ${ }^{21,24,27}$ Eventually, as most erythrocytes were trapped in the fibrin meshwork, the clot was formed and the blood tended to be solidified. The transformation led to 
$\mathrm{Xu}, \mathrm{Lin}$, and Fu: Optical coherence tomography to investigate optical properties...

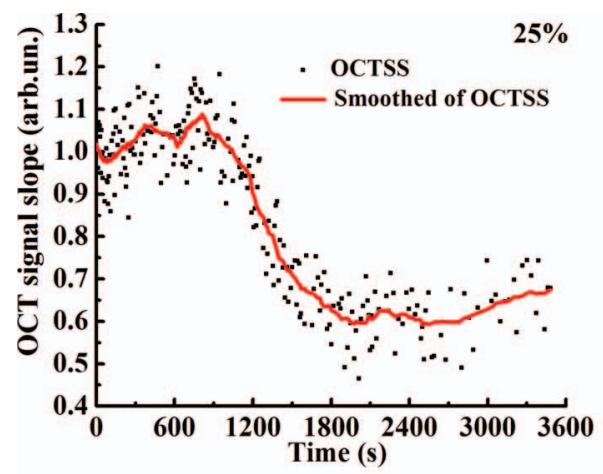

(a)

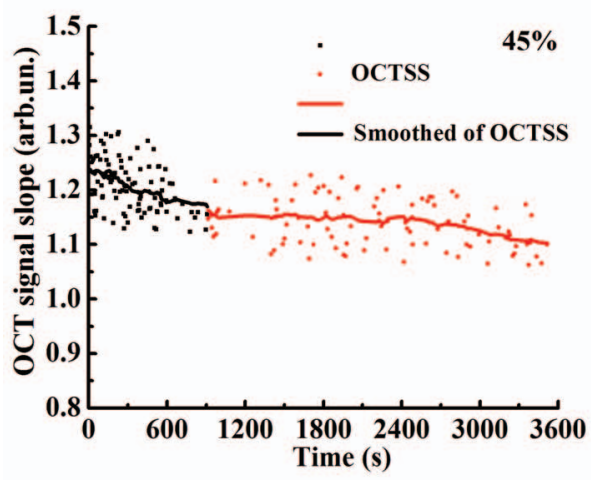

(c)

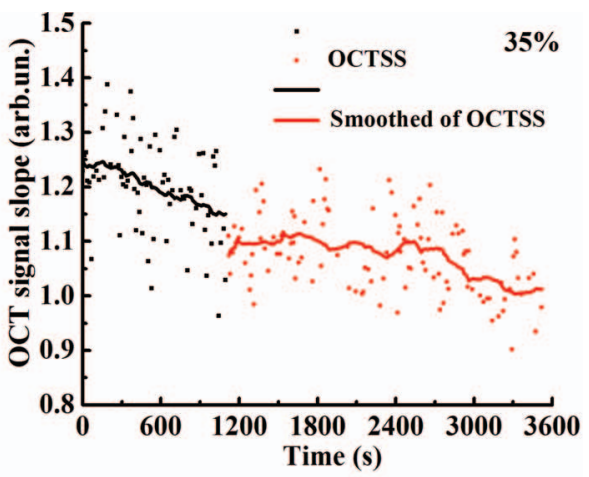

(b)

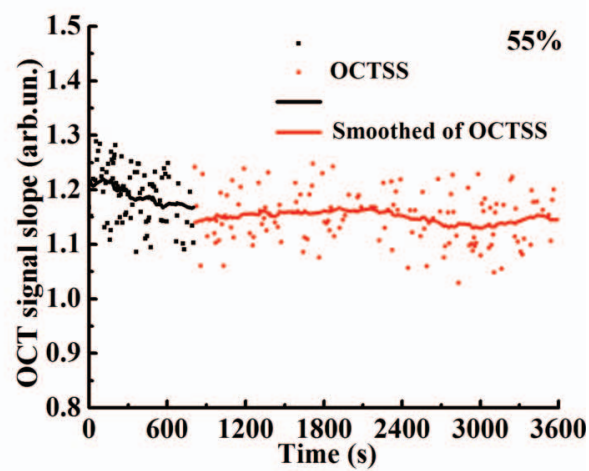

(d)

Fig. 5 OCT signal slope as a function of time obtained from coagulating blood of (a) $25 \%$, (b) $35 \%$, (c) $45 \%$, and (d) $55 \%$ hematocrit. The OCT signal slope decreased with time for all blood samples. Changes in OCTSS decreased with the increase of hematocrit. The coagulation process of the blood at $25 \%$ was abnormal.

the corresponding $1 / e$ light penetration depth at this moment, i.e., the time point $t_{c}$ to be stabilized and smaller changes in the OCT signal slope. There was not much apparent variation from the OCT signal slope given in Fig. 3(a) compared with the results from the 1/e light penetration depth in Fig. 3(b) during the process of blood coagulation. Although the variation in both OCTSS and $d_{1 / e}$ against time was divided into two stages, the stage point and the variation rate between the two differed. The stage point for variation in $d_{1 / e}$ was estimated to be $395 \mathrm{~s}$ rather than $1100 \mathrm{~s}$ for that in the OCTSS. In addition, changes in $d_{1 / e}$ at the first stage were much stronger and faster than that in OCTSS. This may be due to that $d_{1 / e}$ is more sensitive to the scattering changes (relatively multiple scattering light) than the OCTSS (relatively least scattering light). ${ }^{21}$

Figures 5 and 6 , respectively, demonstrate the values of OCTSS and $d_{1 / e}$ acquired from blood samples at different HCTs of $25 \%, 35 \%, 45 \%$, and $55 \%$ as a function of time. For comparison, the values for the blood sample of $35 \%$ were also included as Figs. 5(b) and 6(b). Similar to the blood of 35\%, the OCTSS for the other three blood samples decreased with time and totally reduced by $47.0 \%, 15.0 \%, 13.7 \%$, and $8.5 \%$ for the blood samples at $25 \%, 35 \%, 45 \%$, and $55 \%$ after 60 min coagulation. The variations in the OCTSS against time could also be divided into two stages: the point was at approximately $900 \mathrm{~s}$ for the blood samples of $45 \%$ [Fig. 5(c)] and $800 \mathrm{~s}$ for 55\% [Fig. 5(d)]. The results indicated that a decrease in the OCTSS during the first stage was faster than that during the second stage for $45 \%$ and $55 \%$, as well as $35 \%$.

Two distinct stages of the evolution of $d_{1 / e}$ were also individualized for the samples at 45\% [Fig. 6(c)] and 55\% [Fig. 6(d)] on each curve: 1. a strong increase in $d_{1 / e}$, corresponding to the liquid-gel transition of whole blood, and 2. a plateau with small variations. The clotting time $\left(t_{c}\right)$ was approximately 375 $\pm 12 \mathrm{~s}, 455 \pm 11 \mathrm{~s}$, and $865 \pm 47 \mathrm{~s}$ for the blood at HCTs of $35 \%, 45 \%$, and $55 \%$, respectively. Moreover, the parameter $S_{r}$, defined as the slope of $d_{1 / e}$ within the period of time where $d_{1 / e}$ increased dramatically following the induction of blood coagulation, were calculated to characterize dependence of scattering and attenuation properties of the coagulating blood on different hematocrits. In accordance with the increase of blood HCTs from $35 \%, 45 \%$, to $55 \%$, the $S_{r}\left(\times 10^{-4} \mathrm{~mm} / \mathrm{s}\right)$ tended to decrease from $6.0 \pm 0.3,3.7 \pm 0.5$ to $2.3 \pm 0.4$. These results also consistently indicate that the clotting time was longer for the blood of higher HCT than that of lower HCT. ${ }^{35}$ Considering the parameters from the variations in $d_{1 / e}$ such as $t_{c}$ and $S_{r}$ had better sensitivity and smaller standard deviation than the slopes of OCTSS versus time, they seemed to be more suitable parameters for detecting blood coagulation in in vitro test.

Unlike the blood samples at HCTs of $35 \%, 45 \%$, and $55 \%$, the blood at $25 \%$ had different variation evolution in OCTSS [Fig. 5(a)] and $d_{1 / e}$. There were three stages on each curve: 


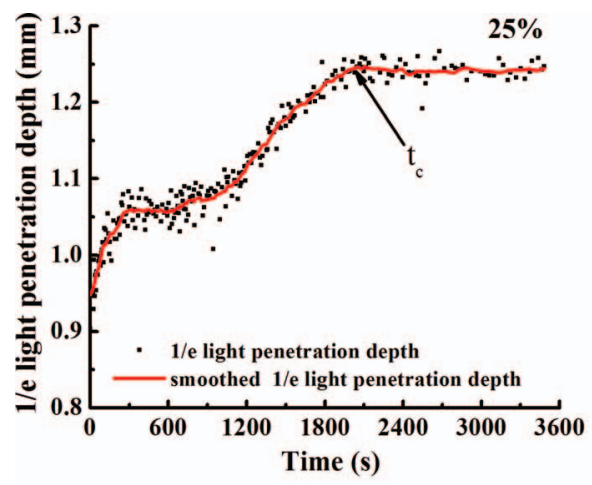

(a)

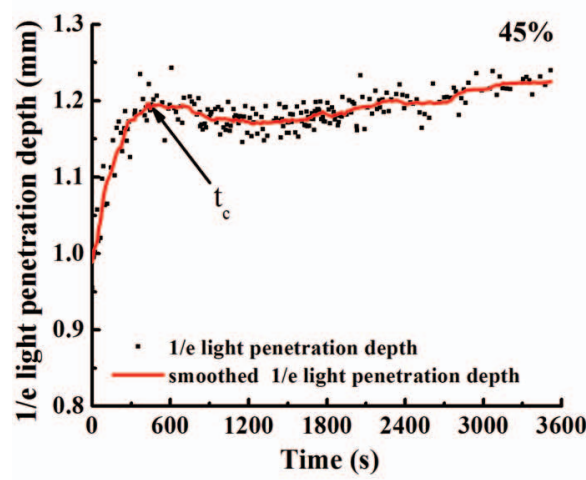

(c)

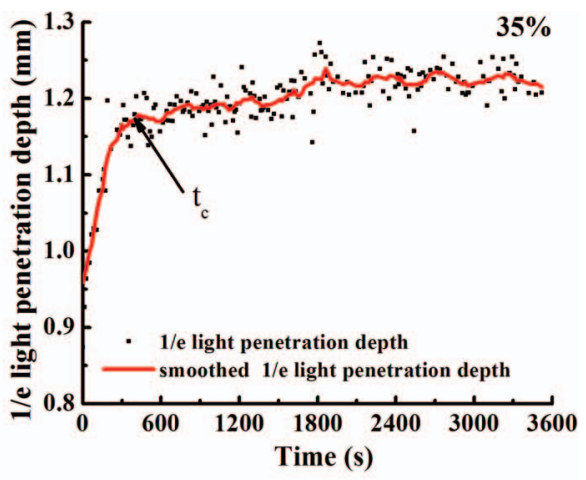

(b)

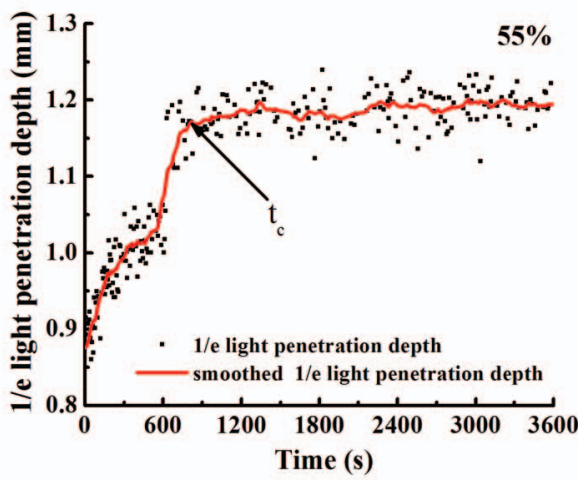

(d)

Fig. 6 1/e light penetration depth as a function of time obtained from coagulating blood of (a) $25 \%$, (b) $35 \%$, (c) $45 \%$, and (d) $55 \%$ hematocrit. The clotting time $\left(t_{c}\right)$ was indicated as an arrow symbol. The clotting time was increased with the increase of hematocrit except for the blood at HCT $=25 \%$.

regarding OCTSS 1. a plateau (0 to $900 \mathrm{~s}$ ), 2. a rapid decrease in OCTSS (900 to $1800 \mathrm{~s}$ ), and 3. another plateau (1800 to $3600 \mathrm{~s})$. The curve regarding $d_{1 / e}$ [Fig. 6(a)] included the first stage ( 0 to $282 \mathrm{~s}$ ) with strong variations, the second stage (283 to $1969 \mathrm{~s}$ ) with moderate variations, and a plateau with no or very small variations (1969 to $3600 \mathrm{~s}$ ). The clotting time was approximately $1969 \mathrm{~s}$ that was largely delayed compared with that for the blood at $35 \%, 45 \%$, or $55 \%$. These results indicated a slower coagulation for the blood at very low HCT. The abnormality in optical properties of the $25 \%$ blood, which is consistent with the previous findings, ${ }^{8}$ resulted from the blood at an abnormal hematocrit. The hematocrit, defined as the ratio of the volume of packed red blood cells to the volume of whole blood, is normally $40 \%$ to $50 \%$ in men and $35 \%$ to $45 \%$ in women. The hematocrit changes in certain circumstances or disease, for patients with blood disorders, during dialysis, in an emergency room environment, and during surgery. For example, if the HCT is higher, he or she may have high red blood cell proliferation problem. On the contrary, it can be seen in people who suffer from anemia. Recent studies have revealed that blood coagulation is HCT dependent in which patients with an abnormally lower HCT might interfere with the capability of blood coagulation for bleeding, and that with an abnormally higher HCT of the blood is more likely to be associated with the increasing risk with the formation of thrombosis or embolism. ${ }^{36}$ Our results demonstrate that it is possible to recognize abnormality in the coagulation rate by OCT detection.

\section{Conclusions}

In this paper, the OCT technique has demonstrated the efficiency to follow liquid to solid transition during blood clotting. Measurements of OCT signal slope and relative 1/e light penetration depth permitted description of the dynamics of the blood clotting process. OCT parameters such as $t_{c}$ and $S_{r}$ made it possible to describe the temporal sequence of two stages of the blood clotting process and the clot growth rate. Furthermore, the parameters were proposed to discriminate between different hematocrits. Certainly, more studies, such as the establishment of a unified relationship between clot characterization and quantitative OCT parameters, need to be carried out. Future investigations with standardized blood samples and with different blood coagulation protocols, particularly to explore the extrinsic pathway, will provide a more accurate evaluation of the potential of this technique.

\section{Acknowledgments}

This work was supported by research grants from the National Natural Science Foundation of China (Grant No. 30870675) and Zhejiang Provincial Natural Science Foundation.

\section{References}

1. D. L. Hoyert, H. Kung, and B. L. Smith, "Deaths: Preliminary data for 2003," Natl. Vital Stat. Rep. 53(15), 1-48 (2005). 
$\mathrm{Xu}$, Lin, and Fu: Optical coherence tomography to investigate optical properties...

2. S. P. Bajaj and J. H. Joist, "New insights into how blood clots: implications for the use of APTT and PT as coagulation screening tests and in monitoring of anticoagulant therapy," Semin Thromb Hemos 25(4), 407-418 (1999).

3. G. Gravlee, S. Arora, S. Lavender, S. Mills, A. Hudspeth, A. Cordell, R. James, J. Brockschmidt, and J. Stuart, "Predictive value of blood clotting tests in cardiac surgical patients," Ann. Thorac. Surg. 58, 216-221 (1994)

4. C. Schmitt, A. H. Henni, and G. Cloutier, "Characterization of timevarying mechanical viscoelastic parameters of mimicking deep vein thrombi with 2D dynamic elastography," Proc.-IEEE Ultrason. Symp. 10, 1009-1012 (2007).

5. G. Guhr, R. Brünig, and H. Schmidt, "Monitoring changes of viscoelasticity during blood coagulation with acoustic sensors," IEEE Int. Frequency Control Symposium 2007, Joint with the 21st Eur. Frequency and Time Forum, pp. 577-580 (2007).

6. G. Guhr, R. Kunze, G. Martin, H. Schmidt, M. Weihnacht, S. Gehrisch, and G. Siegert, "Thrombelastography using acoustic sensors," Proc.-IEEE Ultrason. Symp. 10, 544-547 (2006).

7. R. Gatto, E. D'Amico, W. W. Mantulin, E. Gratton, and F. Charbel, "Optical microprobe for blood clot detection," U. S. Patent US2008/0300493A1 (2008).

8. M. Katayama, S. Hoshiko, T. Izumi, Y. Mishima, W. Meyers, and N. Zander, "Analyzing method of a blood coagulation reaction," U. S. Patent US7276376B2 (2007).

9. B. Sqrensen, P. Johansen, K. Christiansen, M. Woelke, and J. Ingerslev, "Whole blood coagulation thrombelastographic profiles employing minimal tissue factor activation," J. Throm. Hae. 1, 551-558 (2003).

10. T. W. Barrowcliffe, M. Cattaneo, G. M. Podda, P. Bucciarelli, Fer. Lussana, A. Lecchi, C. H. Toh, H. C. Hemker, S. B. Guin, J. Ingerslev, and B. Sqrensen, "New approaches for measuring coagulation," Haemophilia 12(3), 76-81 (2006).

11. J. Gennisson, S. Lerouge, and G. Cloutier, "Assement by transient elastography of the viscoelastic properties of blood during clotting," Ultrasound Med. Biol. 32(10), 1529-1537 (2006).

12. C. Huang, S. Wang, and S. Member, "Assessment of blood coagulation under various flow conditions with ultrasound backscattering," IEEE Trans. Biomed. Eng. 54(12), 2223-2230 (2007).

13. R. Libgot-Callé, F. Ossant, Y. Gruel, P. Lermusiaux, and F. Patat, "High frequency ultrasound device to investigate the acoustic properties of whole blood during coagulation," Ultrasound Med. Biol. 34(2), 252264 (2008).

14. R. Calléa, C. Plag, F. Patat, and F. Ossant, "Interest of the attenuation coefficient in multiparametric high frequency ultrasound investigation of whole blood coagulation process," J. Acoust. Soc. Am. 125(1), 530538 (2009).

15. A. M. K. Nilsson, G. W. Lucassen, W. Verkruysse, S. Andersson-Engels, and M. J. C. van Gemert, "Changes in optical properties of human whole blood in vitro due to slow heating," Photochem. Photobiol. 65(2), 366373 (1997).

16. J. K. Barton, D. P. Popok, and J. F. Black, "Thermal analysis of blood undergoing laser photocoagulation," IEEE J. Sel. Top. Quantum Electron 7(6), 936-943 (2001).

17. J. F. Blacky and J. K. Barton, "Chemical and structural changes in blood undergoing laser photocoagulation," Photochem. Photobiol. 80, 89-97 (2004).

18. D. Huang, E. A. Swanson, C. P. Lin, J. S. Schuman, W. G. Stinson, W. Chang, M. R. Hee, T. Flotte, K. Gregory, C. A. Puliafito, and J. G.
Fujimoto, "Optical coherence tomography," Science 254(5035), 11781181 (1991).

19. Z. Chen, T. E. Milner, S. Srinivas, X. Wang, A. Malekafzali, M. J. C. van Gemert, and J. S. Nelson, "Noninvasive imaging of invivo blood flow velocity using optical doppler tomography," J. Opt. Soc. Am. 22(14), 1119-1121 (1997)

20. M. Brezinski, K. Saunders, C. Jesser, X. Li, and J. Fujimoto, "Index matching to improve optical coherence tomography imaging through blood," Circulation 103, 1999-2003 (2001).

21. V. V. Tuchin, X. Xu, and R. K. Wang, "Dynamic optical coherence tomography in studies of optical clearing, sedimentation, and aggregation of immersed blood," Appl. Opt. 41(1), 258-271 (2002).

22. L. Yu and Z. Chen, "Doppler variance imaging for three-dimensional retina and choroid angiography," J. Biomed. Opt. 15(1), 016029 (2010).

23. M. Y. Kirillin, A. V. Priezzhev, V. V. Tuchin, R. K. Wang, and R. Myllyl, "Effect of red blood cell aggregation and sedimentation on optical coherence tomography signals from blood samples," J. Phys. D 38, 2582-2589 (2005).

24. X. Xu, R. K. Wang, J. B. Elder, and V. V. Tuchin, "Effect of dextraninduced changes in refractive index and aggregation on optical properties of whole blood," Phys. Med. Biol. 48, 1205-1221 (2003).

25. R. K. Wang and V. V. Tuchin, "Optical tissue clearing to enhance imaging performance for OCT," Chapter 2 in Optical Clearing of Tissues and Blood, pp. 854-886, SPIE Press, Bellingham, WA (2005).

26. N. V. Iftimia, D. X. Hammer, C. E. Bigelow, D. I. Rosen, T. Ustun, A. A. Ferrante, D. Vu, R. D. Ferguson, and A. A. F. D. Ustun, "Toward noninvasive measurement of blood hematocrit using spectral domain low coherence interferometry and retinal tracking," Opt. Express 14(8), 3377-3388 (2006).

27. X. Xu, L. Yu, and Z. Chen, "Optical clearing of flowing blood using dextrans with spectral domain optical coherence tomography," J. Biomed. Opt. 13(2), 021107 (2008).

28. X. Xu, L. Yu, and Z. Chen, "Effect of erythrocyte aggregation on hematocrit measurement using spectral-domain optical coherence tomography," IEEE Trans. Biomed. Eng. 55(12), 2753-2758 (2008).

29. X. Xu, Y. C. Ahn, and Z. Chen, Feasibility of Doppler variance imaging for red blood cell aggregation characterization, J. Biomed. Opt. 14(6), 060507 (2009).

30. X. Xu, L. Yu, and Z. Chen, "Velocity variation assessment of red blood cell aggregation with spectral domain doppler optical coherence tomography," Ann. Biomed. Eng. 38(10), 3210-3217 (2010).

31. G. Mohamad, V. V. Tuchin, and K. V. Larin, "Depth-resolved monitoring of glucose diffusion in tissues by using optical coherence tomography," Opt. Lett. 31(15), 2314-2316 (2006).

32. X. Xu and Q. Zhu, "Sonophoretic delivery for contrast and depth improvement in skin optical coherence tomography," IEEE J. Sel. Top. Quantum Electron. 14(1), 56-61 (2008).

33. X. Xu, Q. Zhu, and C. Sun, "Combined effect of ultrasound-SLS on skin optical clearing," IEEE Photonics Technol. Lett. 20(24), 21172119 (2008)

34. D. P. Popescu and M. G. Sowa, "Characteristics of time-domain optical coherence tomography profiles generated from blood-saline mixtures," Phys. Med. Biol. 54(15), 4759-4775 (2009).

35. C. Huang and S. Wang, "Characterization of blood properties from coagulating blood of different hematocrits using ultrasonic backscatter and attenuation," Jpn. J. Appl. Phys. 45, 7191-7196 (2006).

36. H. Ujiie, M. Kawasaki, Y. Suzuki, and M. Kaibara, "Influence of age and hematocrit on the coagulation of blood," J. Biorheology 23, 111-114 (2009). 